\title{
Application of Somatostatin, Chemotherapy Combined with TAE in Heterogeneous Glucagonoma Presented with Necrolytic Migratory Erythema
}

This article was published in the following Dove Press journal: OncoTargets and Therapy

\author{
Chaoyong Shen ${ }^{1, *}$ \\ Jiaoxue $\mathrm{He}^{2, *}$ \\ Xiaoyan Le ${ }^{2}$ \\ Linmao Zheng ${ }^{3}$ \\ Dan $\mathrm{Cao}^{4}$
}

'Department of Gastrointestinal Surgery, West China Hospital, Sichuan University, Chengdu 6I004I, Sichuan, People's Republic of China; ${ }^{2}$ Department of Oncology, Wenjiang District People's Hospital, Chengdu, Sichuan, People's Republic of China; ${ }^{3}$ Department of Pathology, West China Hospital, Sichuan University, Chengdu 61004I, Sichuan, People's Republic of China; ${ }^{4}$ Department of Abdominal Oncology, Cancer Center of West China Hospital, Sichuan University, Chengdu 6I004I, Sichuan, People's Republic of China

*These authors contributed equally to this work
Correspondence: Dan Cao Department of Abdominal Oncology, Cancer Center of West China Hospital, Sichuan University, Chengdu 61004I, Sichuan, People's Republic of China

Tel +86-28-18980605963

Fax +86-28-85422589

Email caodan316@I63.com

\begin{abstract}
Glucagonoma, a rare neuroendocrine tumor of the pancreas, which is often misdiagnosed because of non-characteristic clinical manifestations. In addition, the treatment has not been well established for this disease so far. We here report a case of glucagonoma previous misdiagnosed as recurrent erythema. In this case, necrolytic migratory erythema was the main clinical manifestation, and he received surgical resection after admission although with liver metastasis. Postoperative pathological results showed that the heterogeneity of proliferative index in primary (Ki-67: 5 10\%) and metastatic (Ki-67: 25 30\%) tumors were obviously observed. One month postoperatively, abdominal CT and MRI showed multiple liver metastasis (type III) again. Interestingly, the skin rash was obviously improved after treatment with somatostatin combined with chemotherapy (octreotide, temozolomide and capecitabine). Subsequently, the patient received transarterial embolization (TAE). Up to now, no progression was noted for liver metastasis. Due to its rarity, clinical diagnosis is challenging; thus, further understanding of the disease by clinicians is helpful for early diagnosis and treatment, so as to improve the prognosis of patients.
\end{abstract} Keywords: glucagonoma, necrolytic migratory erythema, somatostatin, chemotherapy, TAE

\section{Introduction}

Glucagonoma is an extremely uncommon neuroendocrine tumor arising from pancreatic islet cells. The common clinical manifestation of glucagonoma is the necrolytic migratory erythema, which is unspecific, and may be misdiagnosed as eczema, pemphigus, etc. ${ }^{1-7}$ In order to diagnose glucagonoma, it is necessary to improve the detection of glucagon. Pathological grading of glucagonoma should be made according to the expression of Ki-67, and the treatment scheme for different grades is quite different. ${ }^{8}$ Meanwhile, there is a need to perform somatostatin receptor (SSTR) detection, as well as the Ga68PET/CT should be conducted if necessary to estimate distant metastasis. ${ }^{9}$

In terms of treatment, biotherapy can be given to enhance tumor control and relieve clinical symptoms during chemotherapy or targeted treatment. ${ }^{10}$ Therefore, it is urgent to improve the diagnosis rate and treatment efficiency of glucagonoma due to the high misdiagnosis rate of glucagonoma. Additionally, somatostatin combined with chemotherapy in the treatment of glucagonoma is rare, especially for those with liver metastasis postoperatively. Thus, we here report a case diagnosed as glucagonoma who presented with necrolytic migratory erythema and was successfully treated with somatostatin combined with chemotherapy. 


\section{Case Report}

A 46-year-old male patient was admitted to the hospital who presented with systemic repeated erythema with pruritus and local necrosis (Figure 1). Thirteen months ago, this patient was diagnosed as eczema or pemphigus in other hospital; the symptoms improved after corresponding treatment, but the erythema still occurred repeatedly. During the course of treatment, abdominal enhanced MRI was performed at outside hospital, which showed that a mass occupied the tail of pancreas with multiple metastases of liver. Subsequently, the patient went to our hospital for abdominal CT examination again, and similar imaging results were found (Figure 2). Therefore, the laparoscopic pancreaticocaudal resection + splenectomy + left hemihepatectomy + right liver tumor resection + cholecystectomy + portal vein repair were performed on April 1, 2019. Complete resection of the primary tumor and hepatic metastases was obtained. The results of immunohistochemistry in primary tumor showed that the expression of PCK, EMA (weak), CgA, Syn, CD56, Vim and glucagon was positive, and the positive rate of $\mathrm{Ki}-67$ (MIB-1) was 5 10\% (Figure 3). Tumor cells in multiple liver metastases were observed, and the highest positive rate of Ki67 (MIB-1) in the live lesions range from 25\% to $30 \%$. Postoperatively, the skin rash gradually improved. After 10 days, erythema appeared again, accompanied by pruritus and local necrosis. Results of blood sampling showed that serum glycosylated antibody 125 was increased with a value of $62.98 \mathrm{u} / \mathrm{mL}$, as well as the fasting blood glucose was slightly increased again with a value of $6.51 \mathrm{mmol} / \mathrm{l}$. As such, we supplemented the immunohistochemistry test, and the result indicated that the expression of SSTR2 was positive. Abdominal enhanced CT and upper abdomen MRI showed multiple liver nodules (May 2019). Thus, the first cycle of chemotherapy was given on May 11, 2019 (capecitabine: 1000mg BID day 1-14 + temozolomide 200mg QD day 10-14, 4 weeks as a cycle; octreotide 40mg QD day 1, 4 weeks as a cycle). On the 27th day of treatment, the skin lesions on the face of the patient were disappeared, and pigmentation remained on the skin of the legs. Up to now, this patient has received this therapeutic regimen for 5 cycles. On October 10, 2019, the patient underwent transarterial embolization with local anesthesia. Follow up to


Figure I Representative photograph of the patient's erythema at presentation before (A) and after (B) surgery. The skin erythema reappeared and Comment: gradually become serious at 10 days after surgery $(\mathbf{C}-\mathbf{E})$. The skin rash on the face of the patient has disappeared, and the pigmentation remained on the skin of the legs on the 27 th day of treatment with octreotide+chemotherapy $(\mathbf{F}-\mathbf{H})$. 

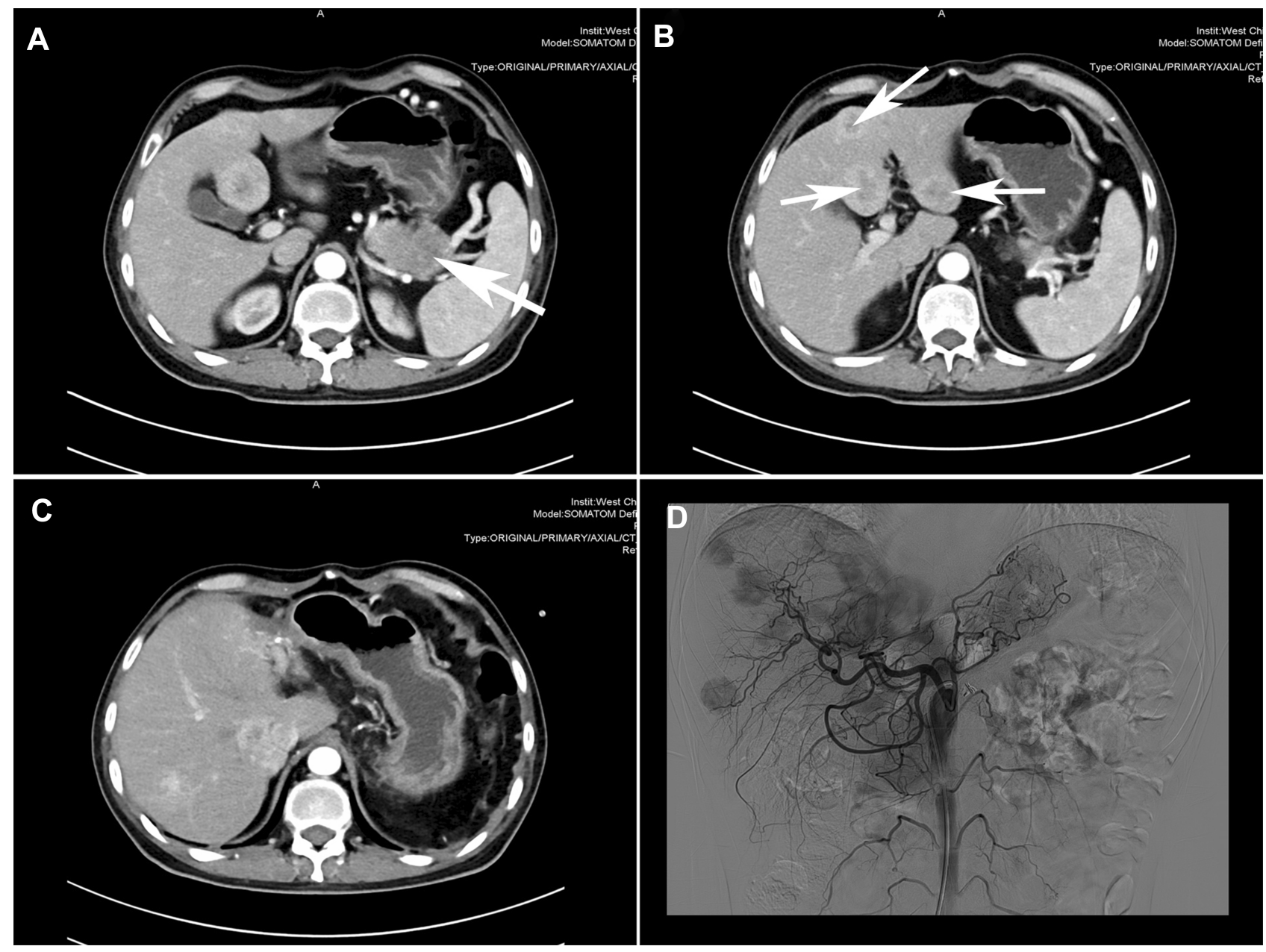

Figure 2 On March 25, 2019, abdominal CT showed a mass $(4.2 * 3.5 \mathrm{~cm})$ in the tail of pancreas, and multiple liver nodules were observed (A, B). Abdominal enhanced CT showed multiple liver nodules (C, May 2019). On October 10, 2019, the patient underwent transcatheter arterial chemoembolization with local anesthesia (D).

October 31, 2019, no tumor progression was observed for this patient. Finally, the written informed consent has been provided by this patient, and the study was approved by the Institutional Review Board of West China Hospital.

\section{Discussion}

Glucagonoma accounts for less than $10 \%$ of pancreatic neuroendocrine tumors, with an annual prevalence of $1 /$ 20 million. ${ }^{11}$ Tumor originating from Islet alpha cells, also known as $\alpha$ cell tumor, most of which are malignant and often metastasized at early stage. The diagnosis of glucagonoma requires the discovery of $\alpha$ cell secretory granules by electron microscopy, and/or immunohistochemistry showed that glucagon, $\mathrm{CgA}$ and Syn were positive. ${ }^{12}$ Generally, the normal range of glucagon is $25 \sim 250 \mathrm{pg}$ / $\mathrm{mL}$; when suffering from glucagonoma, it usually exceeds $500-1000 \mathrm{pg} / \mathrm{mL}$. However, it is insufficient to diagnose glucagonoma promptly in clinic, and misdiagnosis often occurs. Therefore, this needs to be highly valued by clinicians. Document data displayed that a total of $49.2 \%$ of patients had metastases upon diagnosis. ${ }^{7}$ The most common sites of metastasis were liver, local lymph node, followed by bone, lung and adrenal gland..$^{7,13,14}$ It is often necessary to localize glucagonoma by CT or MRI. The sensitivity of somatostatin receptor radionuclide imaging (Ga68 PET/CT) is higher than other imaging methods, and it has been recommended as the first choice for localization and grading of glucagonoma.

The neuroendocrine tumors were graded into four types following the newly WHO classification. And the definition of each grade is as follows: pancreatic endocrine tumor G1 $(\mathrm{Ki}-67 \leq 2 \%)$, pancreatic endocrine tumor G2 $(3 \%<\mathrm{Ki}-67 \leq$ $20 \%$ ), pancreatic endocrine tumor G3 (highly differentiated, Ki-67> 20\%), neuroendocrine cancer G3 (NEC, poorly differentiated, Ki-67 > 20\%). ${ }^{15}$ Although the Ki-67 index in the primary tumor of this patient is not very high, it has 

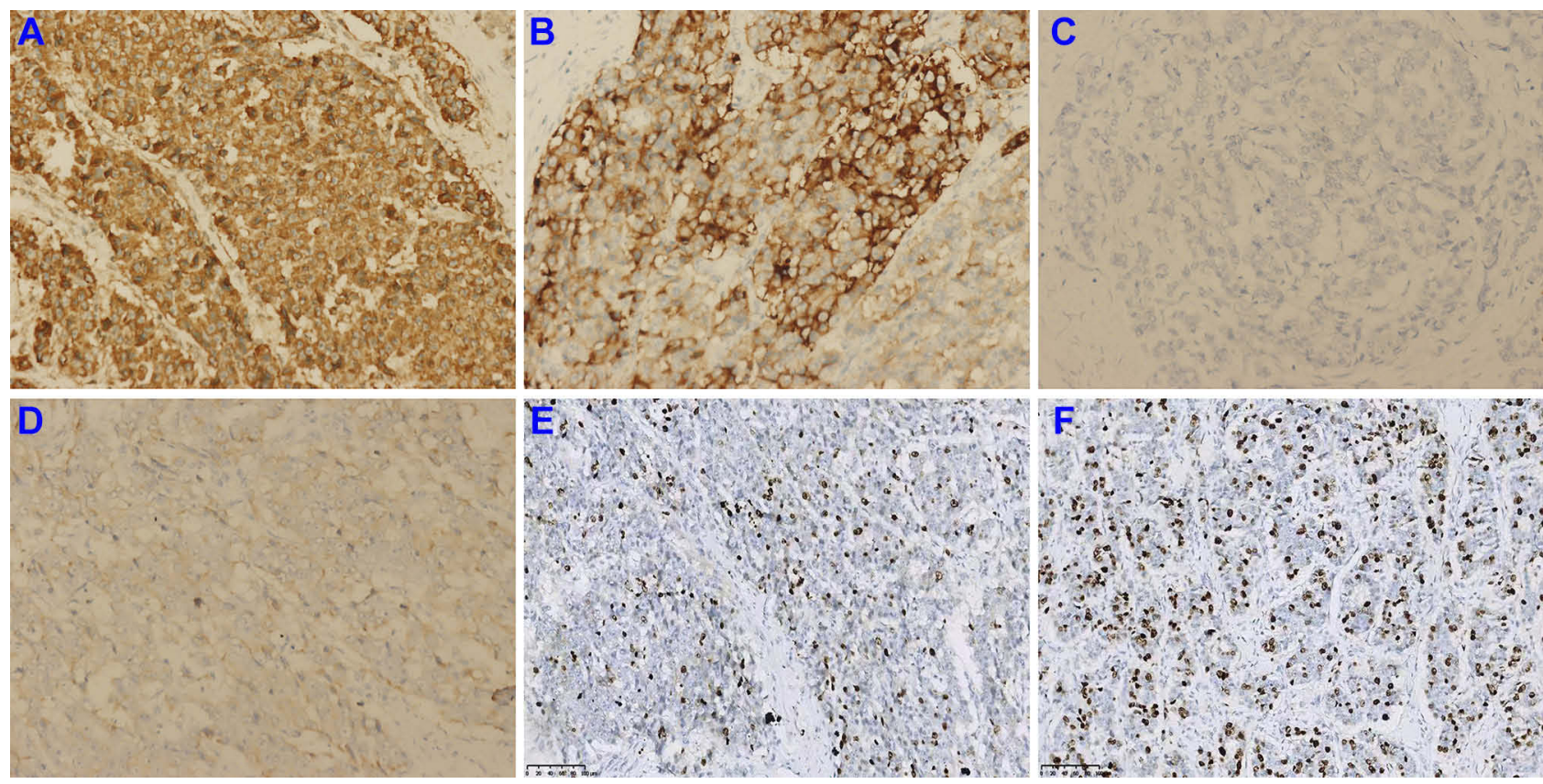

Figure 3 Positive expression of CgA (A), Syn (B), SSTR2 (C), and glucagon (D) in primary tumor tissue. The Ki-67 index was 5 10\% (E) and 25 30\% (F) in the primary tumor and liver metastases, respectively.

a high invasiveness with a tendency of distant metastasis. Interestingly, we observed significant heterogeneity of Ki-67 index in metastatic and primary tumors.

Surgery is currently the only method to cure glucagonoma. Complete tumor resection with local lymph nodes dissection should be performed for the local glucagonoma. ${ }^{16}$ In addition, simultaneous or staged resection can be performed for the resectable liver metastases. Previously, several treatment options can be used in patients with liver metastasis, such as TAE, radiofrequency ablation and tumor reduction. ${ }^{17,18}$ To some extent, it can improve the prognosis of patients. The hepatic metastases of this patient had abundant blood supply; thus, he received transarterial embolization (TAE). Generally, multi-disciplinary comprehensive treatment is the main treatment for neuroendocrine tumors at an advanced stage, ${ }^{19}$ among which internal medicine treatment is an important part. The combination chemotherapy scheme based on streptozotocin is recommended for G1/G2 pancreatic neuroendocrine tumor (p-NET), and the combination chemotherapy scheme based on temozolomide is recommended for G1/G2/ G3 p-NET.

Since streptozotocin is not listed in China, temozolomide is commonly used as a single drug or combined with capecitabine for chemotherapy. ${ }^{20}$ In p-NET with high proliferative activity, this scheme may also have some therapeutic effect. ${ }^{21}$ EP and IP chemotherapy schemes are recommended for the treatment of G3 p-NEC. In patients with glucagonoma, the expression of SSTR should be detected. Somatostatin analogues, such as octreotide and lanreotide, can be combined with somatostatin receptor type 2 and 5 receptors to block the release of glucagon and other hormones, and thus control related symptoms. ${ }^{22}$ Furthermore, somatostatin drugs also have the effect of anti-tumor proliferation. PROMID trial showed that the prognosis of neuroendocrine midgut tumors has been significantly improved after the application of somatostatin analogues. ${ }^{23}$ In addition, the peptide receptor radionuclide therapy (PRRT) plays an important role in treating these tumors. The combination of radionuclide labeled somatostatin drugs and tumor with high expression of somatostatin receptor can achieve the purpose of tumor treatment, and improve the prognosis of patients. At present, a variety of such drugs have been used in the clinic, such as $111 \mathrm{In}$ DTPAOC, 90Y-DOTALAN, 90Y-DOTATOC, 90YDOTATATE, and 177Lu-DOTATATE, etc. ${ }^{24}$ Therefore, PRRT can be tried after chemotherapy failure for those with high proliferative activity and SSTR positive.

\section{Conclusions}

Clinically, glucagonoma is often misdiagnosed due to noncharacteristic clinical symptoms, such as rash, hyperglycemia. Combined with this report, early recognition, diagnosis and treatment of glucagonoma can improve treatment efficiency. For patients with glucagonoma, the multidisciplinary treatment mode with somatostatin controlling 
functional symptoms and TAE reducing tumor burden of liver metastasis can improve the prognosis of patient to a certain extent.

\section{Informed Consent Statement}

The written informed consent has been provided by the patient to have the case details and any accompanying images published.

\section{Acknowledgments}

We are very grateful to the staff at the Department of Abdominal Oncology, Cancer Center of West China Hospital, Sichuan University for their kind work in data collection.

\section{Author Contributions}

CD conceived and designed the study. SCY and HJX drafted the manuscript. LXY and ZLM provided supervision. CD critically revised the manuscript for important intellectual content. All authors made substantial contributions to conception and design, acquisition of data, or analysis and interpretation of data; took part in drafting the article or revising it critically for important intellectual content; gave final approval of the version to be published; and agree to be accountable for all aspects of the work.

\section{Disclosure}

The authors declare that they have no competing interests related to this work.

\section{References}

1. Gao Y, Wang C, Gao Y, et al. Glucagonoma syndrome with serous oligocystic adenoma: a rare case report. Medicine. 2017;96(43):e8448. doi:10.1097/MD.0000000000008448

2. Tolliver S, Graham J, Kaffenberger BH. A review of cutaneous manifestations within glucagonoma syndrome: necrolytic migratory erythema. Int $J$ Dermatol. 2018;57(6):642-645. doi:10.1111/ ijd.2018.57.issue-6

3. Toberer F, Hartschuh W, Wiedemeyer K. Glucagonoma-associated necrolytic migratory erythema: the broad spectrum of the clinical and histopathological findings and clues to the diagnosis. Am J Dermatopathol. 2019;41 (3):e29-e32. doi:10.1097/DAD.0000000000001219

4. Wang ZX, Wang F, Zhao JG. Glucagonoma syndrome with severe erythematous rash: a rare case report. Medicine. 2019;98(37):e17158. doi:10.1097/MD.0000000000017158

5. He S, Zeng W, Geng S, Jia J. Glucagonoma syndrome with atypical necrolytic migratory erythema. Indian J Dermatol Venereol Leprol. 2019.

6. Guilbaud T, Berbis P, Birnbaum DJ. Glucagonoma with paraneoplasic dermatitis: diagnosis and management. J Gastrointest Surg. 2019. doi:10.1007/s11605-019-04267-0
7. Song X, Zheng S, Yang G, et al. Glucagonoma and the glucagonoma syndrome. Oncol Lett. 2018;15(3):2749-2755. doi:10.3892/ol.2017. 7703

8. Pelosi G, Zamboni G, Doglioni C, et al. Immunodetection of proliferating cell nuclear antigen assesses the growth fraction and predicts malignancy in endocrine tumors of the pancreas. Am J Surg Pathol. 1992;16(12):1215-1225. doi:10.1097/00000478-199212000-00008

9. Sahoo MK, Gupta S, Singh I, Pahwa S, Durgapal P, Bal CS. Necrolytic migratory erythema associated with glucagonoma syndrome diagnosed by (6)(8)Ga-DOTANOC PET-CT. Asia Pac J Clin Oncol. 2014;10(2):190-193. doi:10.1111/ajco.12048

10. Dimitriadis GK, Weickert MO, Randeva HS, Kaltsas G, Grossman A. Medical management of secretory syndromes related to gastroenteropancreatic neuroendocrine tumours. Endocr Relat Cancer. 2016;23 (9):R423-R436. doi:10.1530/ERC-16-0200

11. Ferrarese A, Borello A, Gentile V, et al. Meso-pancreatectomy for pancreatic neuroendocrine tumor. Int J Surg. 2014;12(Suppl 1):S123S125. doi:10.1016/j.ijsu.2014.05.031

12. Teixeira RC, Nico MM, Ghideti AC. Necrolytic migratory erythema associated with glucagonoma: a report of 2 cases. Clinics (Sao Paulo). 2008;63(2):267-270. doi:10.1590/S1807-59322008000200016

13. Gutierrez V, Cobo M, Olea D, et al. Glucagonoma with two pancreatic masses and pulmonary metastases as debut of MEN-1. Clin Transl Oncol. 2007;9(10):674-677. doi:10.1007/s12094-007-0121-5

14. Tseng HC, Liu CT, Ho JC, Lin SH. Necrolytic migratory erythema and glucagonoma rising from pancreatic head. Pancreatology. 2013;13(4):455-457. doi:10.1016/j.pan.2013.03.011

15. Scoazec JY, Couvelard A. Classification of pancreatic neuroendocrine tumours: changes made in the 2017 WHO classification of tumours of endocrine organs and perspectives for the future. Ann Pathol. 2017;37(6):444-456. doi:10.1016/j.annpat.2017.10.003

16. Cao X, Wang X, Lu Y, et al. Spleen-preserving distal pancreatectomy and lymphadenectomy for glucagonoma syndrome: a case report. Medicine. 2019;98(38):e17037. doi:10.1097/MD.0000000000017037

17. Poggi G, Villani L, Bernardo G. Multimodality treatment of unresectable hepatic metastases from pancreatic glucagonoma. Rare Tumors. 2009;1(1):e6. doi:10.4081/rt.2009.e6

18. Fujimoto M, Ueda Y, Amari M, et al. Long-time survival case of glucagonoma metastatic to the liver treated with TAE therapy. Nihon Naika Gakkai Zasshi. 1999;88(7):1329-1331. doi:10.2169/naika.88.1329

19. Gunavathy M, Rohana AG, Norlela S, Nor Azmi K. A ten-year retrospective analysis of gastroenteropancreatic neuroendocrine tumors (GEP-NETs) in Malaysia. Med J Malaysia. 2014;69(3):133-137.

20. Pavel M, O'Toole D, Costa F, et al. ENETS consensus guidelines update for the management of distant metastatic disease of intestinal, pancreatic, bronchial Neuroendocrine Neoplasms (NEN) and NEN of unknown primary site. Neuroendocrinology. 2016;103(2):172-185. doi: $10.1159 / 000443167$

21. Olsen IH, Sorensen JB, Federspiel B, et al. Temozolomide as second or third line treatment of patients with neuroendocrine carcinomas. TheScientificWorldJournal. 2012;2012:170496. doi:10.1100/2012/ 170496

22. Toumpanakis C, Caplin ME. Update on the role of somatostatin analogs for the treatment of patients with gastroenteropancreatic neuroendocrine tumors. Semin Oncol. 2013;40(1):56-68. doi:10. 1053/j.seminoncol.2012.11.006

23. Rinke A, Muller HH, Schade-Brittinger C, et al. Placebo-controlled, double-blind, prospective, randomized study on the effect of octreotide LAR in the control of tumor growth in patients with metastatic neuroendocrine midgut tumors: a report from the PROMID study group. J Clin Oncol. 2009;27(28):4656-4663. doi:10.1200/ JCO.2009.22.8510

24. Gulenchyn KY, Yao X, Asa SL, Singh S, Law C. Radionuclide therapy in neuroendocrine tumours: a systematic review. Clin Oncol (R Coll Radiol). 2012;24(4):294-308. doi:10.1016/j.clon.2011.12.003 


\section{Publish your work in this journal}

OncoTargets and Therapy is an international, peer-reviewed, open access journal focusing on the pathological basis of all cancers, potential targets for therapy and treatment protocols employed to improve the management of cancer patients. The journal also focuses on the impact of management programs and new therapeutic agents and protocols on patient perspectives such as quality of life, adherence and satisfaction. The manuscript management system is completely online and includes a very quick and fair peer-review system, which is all easy to use. Visit http://www.dovepress.com/ testimonials.php to read real quotes from published authors. 